\title{
The Stable Roommates Problem with Globally Ranked Pairs
}

\author{
David J. Abraham, Ariel Levavi, David F. Manlove, and Gregg 0’Malley
}

Abstract. We introduce a restriction of the stable roommates problem in which roommate pairs are ranked globally. In contrast to the unrestricted problem, weakly stable matchings are guaranteed to exist, and additionally, they can be found in polynomial time. However, it is still the case that strongly stable matchings may not exist, and so we consider the complexity of finding weakly stable matchings with various desirable properties. In particular, we present a polynomial-time algorithm to find a rank-maximal (weakly stable) matching. This is the first generalization of an algorithm due to [Irving et al. 06] to a nonbipartite setting. Also, we describe several hardness results in an even more restricted setting for each of the problems of finding weakly stable matchings that are of maximum size, are egalitarian, have minimum regret, and admit the minimum number of weakly blocking pairs.

\section{Introduction}

The Stable RoOmmates problem (SR) [Gale and Shapley 62, Irving 85, Gusfield and Irving 89, Irving and Manlove 02] involves pairing a set of agents, each of whom ranks the others in (not necessarily strict) order of preference. Agents can declare each other unacceptable, in which case they cannot be paired together. Our task is to find a pairing of mutually acceptable agents such that no two agents would prefer to partner each other over those that we prescribed for them.

We represent acceptable pairs by a graph $G=(V, E)$, with one vertex $u \in V$ for each agent, and an edge $\{u, v\} \in E$ whenever agents $u$ and $v$ are mutually

(C) A K Peters, Ltd.

|542-795|/08 \$0.50 per page 
acceptable. A pairing is just a matching $M$ of $G$, i.e., a subset of edges in $E$, no two of which share a vertex. If $\{u, v\} \in M$, we say that $u$ is matched in $M$, and $M(u)$ denotes $v$; otherwise, $u$ is unmatched in $M$. An agent $u$ prefers one matching $M^{\prime}$ over another $M$ if (i) $u$ is matched in $M^{\prime}$ and unmatched in $M$ or (ii) $u$ is matched in $M$ and $M^{\prime}$, and $u$ prefers $M^{\prime}(u)$ to $M(u)$. Similarly, $u$ is indifferent between $M^{\prime}$ and $M$ if (i) $u$ is unmatched in $M^{\prime}$ and $M$ or (ii) $u$ is matched in $M$ and $M^{\prime}$, and $u$ is indifferent between $M^{\prime}(u)$ and $M(u)$.

A matching $M$ is weakly stable if it admits no strongly blocking pair, which is an edge $\{u, v\} \in E \backslash M$ such that $u$ and $v$ prefer $\{\{u, v\}\}$ to $M$. A matching $M$ is strongly stable if it admits no weakly blocking pair, which is an edge $\{u, v\} \in E \backslash M$ such that $u$ prefers $\{\{u, v\}\}$ to $M$, while $v$ either prefers $\{\{u, v\}\}$ to $M$ or is indifferent between them. Finally, a matching is super stable if it admits no edge $\{u, v\} \in E \backslash M$ such that (i) $u$ either prefers $\{\{u, v\}\}$ to $M$, or is indifferent between them, and (ii) $v$ either prefers $\{\{u, v\}\}$ to $M$ or is indifferent between them.

In this work, we introduce and study the STABLE ROOMMATES WITH GLOBALLY-RANKED PAIRS problem (SR-GRP). An instance of SR-GRP is a restriction of SR in which preferences may be derived from a ranking function rank $: E \rightarrow \mathbb{Z}^{+}$. An agent $u$ prefers $v$ to $w$ if $\operatorname{rank}(e)<\operatorname{rank}\left(e^{\prime}\right)$, where $e=\{u, v\}$ and $e^{\prime}=\{u, w\}$. Similarly, agent $u$ is indifferent between $v$ and $w$ if $\operatorname{rank}(e)=\operatorname{rank}\left(e^{\prime}\right)$.

Before giving our motivation for studying this restriction, we introduce some additional notation. We define $E_{i}$ to be the set of edges with rank $i$, and $E_{\leq i}$ to be the set $E_{1} \cup E_{2} \cup \cdots \cup E_{i}$. Additionally, let $n=|V|$ be the number of agents and $m=|E|$ the number of mutually acceptable pairs. Without loss of generality, we assume that the maximum edge rank is at most $m$. Also, we make the standard assumption in the study of stable matching problems that the adjacency list for a vertex is given in order of preference/rank.

\section{I.I. Motivation}

In several real-world settings, agents have restricted preferences that can be represented by the SR-GRP model. A pairwise kidney-exchange market [Roth et al. 04, Roth et al. 05, Abraham et al. 07a] is one such setting. Here, patients with terminal kidney disease obtain compatible donors by swapping their own willing but incompatible donors. We can model the basic market by constructing one vertex for each patient, and an undirected edge between any two patients indicating that the incompatible donor for one patient is compatible with the other patient, and vice versa. Of course, patients may have different preferences over donors. However, since the expected years of life gained from a transplantation is 
similar among all compatible kidneys, the medical community has suggested that patient preferences should be binary/dichotomous [Gjertson and Cecka 00, Delmonico 04], i.e., patients are indifferent between all compatible donors. Binary preferences are a special case of preferences induced by a global ranking, since all edges have the same rank.

In repeated kidney-exchange markets, swaps are conducted periodically, with patients entering and leaving the market over time. When two (patient, donor) pairs are matched with each other (in order to swap donors), doctors are not certain whether the swap can occur until expensive last-minute compatibility tests are performed on the donors and patients. If either potential transplantation in the swap is incompatible, the swap is canceled and the two patients must wait for a future match. Note that the probability of a swap being compatible is the same for both patients involved in the swap. Also, doctors can predict the probability of a swap being compatible. Hence patient preferences can be inferred by ranking the potential swaps by their chance of success, which is exactly the preference model of SR-GRP.

A third application arises in peer-to-peer (P2P) networks [Gai et al. 07a, Gai et al. 07b, Lebedev et al. 07, Mathieu 07a, Mathieu 07b]. For example, in a P2P file-sharing network, a given peer may form a preference list over other peers based on the similarity of their interests [Lebedev et al. 07]. In cooperative download applications such as BitTorrent, preference functions may be derived from properties such as download/upload bandwidth, latency, and storage capacity. The "Tit-for-Tat" strategy of BitTorrent can give rise to preference lists for peers that are based on a single global ranking (referred to as a master list in [Irving et al. 08, O'Malley 07]) of peers according to upload capacity. The presence of a global ranking of peers (as opposed to edges) gives rise to a special case of SR-GRP (this can be seen as follows: an SR-GRP instance can be obtained by defining the rank of an edge $\{u, v\}$ to be $\operatorname{rank}(u)+\operatorname{rank}(v)$, where $\operatorname{rank}(w)$ is the rank of agent $w$ in the master list). In fact, peers can have nonunitary capacity in general, so this application actually motivates a many-to-many variant of SR-GRP, called the stable b-matching problem [Cechlárová and Fleiner 05] with globally ranked pairs.

One final real-world setting is described in [Arkin et al. 09]. When colleges pair freshmen roommates, it is infeasible for students to rank each other explicitly. Instead, each student submits a form that describes him- or herself in several different dimensions (e.g., bedtime preference, cleanliness preference). Students can then be represented as points in a multidimensional space, and preferences over other students can be inferred by a distance function. Note that this model [Arkin et al. 09] is a restriction of SR-GRP in that it is impossible to declare another student unacceptable. 


\section{I.2. Preliminary Results}

In order to highlight the generality of the SR-GRP model, we introduce a second restriction of SR called STABLE ROOMMATES WITH GLOBALLY ACYCLIC PREFERENCES (SR-GAP). Instances of SR-GAP satisfy the following characterization test: given an arbitrary instance $I$ of sR with $G=(V, E)$, construct a digraph $P(G)$ containing one vertex $e$ for each edge in $e \in E$ and an arc from $e=\{u, v\} \in E$ to $e^{\prime}=\{u, w\} \in E$ if $u$ prefers $w$ to $v$. For each $e=\{u, v\}$ and $e^{\prime}=\{u, w\}$ in $E$, if $u$ is indifferent between $v$ and $w$, merge vertices $e$ and $e^{\prime}$. Note that a merged vertex may contain several original edge vertices and have self-loops. Instance $I$ belongs to sR-GAP if and only if $P(G)$ is acyclic.

Instances of SR-GRP satisfy the SR-GAP test, since any directed path in $P(G)$ consists of arcs with monotonically improving ranks, and so no cycles are possible. In the reverse direction, given any instance of SR-GAP, we can derive a suitable rank function from a reverse topological sort on $P(G)$, i.e., $\operatorname{rank}(e)<$ $\operatorname{rank}\left(e^{\prime}\right)$ if and only if $e$ appears before $e^{\prime}$. The following proposition is clear.

Proposition I.I. Let I be an instance of SR. Then I is an instance of SR-GRP if and only if $I$ is an instance of SR-GAP.

As well as modeling real-world problems, SR-GRP is an important theoretical restriction of SR. It is well known that SR has two key undesirable properties. First, some instances of SR admit no weakly stable matchings (see, for example, [Gusfield and Irving 89, p. 164]). And second, the problem of finding a weakly stable matching, or proving that no such matching exists, is NP-hard [Ronn 90, Irving and Manlove 02]. It turns out that SR-GRP has neither of these undesirable properties.

Lemma 1.2. Let $G=\left(V, E_{1} \cup \cdots \cup E_{m}\right)$ be an instance of SR-GRP. Then $M$ is a weakly stable matching of $G$ if and only if $M \cap E_{\leq i}$ is a maximal matching of $E_{\leq i}$, for all $i$.

Proof. Let $M$ be a matching that is not weakly stable, and suppose for the sake of a contradiction that $M \cap E_{\leq i}$ is a maximal matching of $E_{\leq i}$ for all $i$. Since $M$ is not weakly stable, it admits a blocking pair $e=\{u, v\}$ with some rank, say $r$. It follows that neither $u$ nor $v$ is matched on $M \cap E_{\leq r}$, and so $M \cap E_{\leq r}$ is not maximal on $E_{\leq r}$, a contradiction.

Let $M$ be a weakly stable matching and suppose for the sake of a contradiction that $r$ is the first rank for which $M \cap E_{\leq r}$ is not maximal on $E_{\leq r}$. Since $M \cap E_{\leq r}$ is not maximal, let $e=\{u, v\}$ be some edge in $E_{r}$ that could be added to 
$M \cap E_{\leq r}$ so that $(M \cup e) \cap E_{\leq r}$ would be a legal matching. Since neither $u$ nor $v$ is matched by $M \cap E_{\leq r}$, and $\{u, v\} \in E_{r}$, it follows that $\{u, v\}$ blocks $M$, giving the required contradiction.

So we can construct a weakly stable matching in $O(n+m)$ time by finding a maximal matching on rank-1 edges, removing the matched vertices, finding a maximal matching on rank-2 edges, and so on.

Strongly stable matchings are also easy to characterize in SR-GRP.

Lemma 1.3. Let $G=\left(V, E_{1} \cup \cdots \cup E_{m}\right)$ be an instance of SR-GRP. Then $M$ is a strongly stable matching of $G$ if and only if $M \cap E_{i}$ is a perfect matching of $\left\{e \in E_{i}: e\right.$ is not adjacent to any $\left.e^{\prime} \in M \cap E_{<i}\right\}$, for all $i$.

Proof. Let $M$ be a matching such that $M \cap E_{i}$ is a perfect matching of $\{e \in$ $E_{i}: e$ is not adjacent to any $\left.e^{\prime} \in M \cap E_{<i}\right\}$ for all $i$. Suppose for the sake of a contradiction that $M$ is not strongly stable. It follows that $M$ admits a blocking pair $\{u, v\}$, where without loss of generality, we assume that $u$ prefers $v$ to $M(u)$ and $v$ either prefers $u$ to $M(v)$ or is indifferent between them.

Let $r=\operatorname{rank}(\{u, v\})$ and consider $\left\{e \in E_{r}: e\right.$ is not adjacent to any $e^{\prime} \in$ $\left.M \cap E_{<r}\right\}$. Note that $v$ is not incident on an edge in $E_{<r}$; otherwise, $v$ would be matched in $M \cap E_{<r}$ and would prefer $M(v)$ to $u$. Also, $u$ is unmatched in $E_{\leq r}$, since $u$ prefers $v$ to $M(u)$. Hence $\{u, v\} \in\left\{e \in E_{r}: e\right.$ is not adjacent to any $\left.e^{\prime} \in M \cap E_{<r}\right\}$, and so $M \cap E_{r}$ is not a perfect matching, giving the required contradiction.

Let $M$ be a strongly stable matching and suppose for the sake of a contradiction that $r$ is the first rank for which $M \cap E_{r}$ is not a perfect matching of $\left\{e \in E_{r}: e\right.$ is not adjacent to any $\left.e^{\prime} \in M \cap E_{<r}\right\}$. Since $M \cap E_{r}$ is not perfect, it leaves some vertex $u$ unmatched, where $\{u, v\} \in E_{r}$, and both $u$ and $v$ are unmatched in $M \cap E_{<r}$. If $v$ is also unmatched in $M \cap E_{r}$, then $\{u, v\}$ is a strongly blocking pair of $M$, giving the required contradiction. Alternatively, if $v$ is matched in $M \cap E_{r}$, then $\{u, v\}$ is a weakly blocking pair, contradicting the assumption that $M$ is strongly stable.

Of course, even $E_{1}$ may not admit a perfect matching, and so strongly stable matchings may not exist. However, we can find a strongly stable matching or prove that no such matching exists in $O(m \sqrt{n})$ time using the maximum matching algorithm of Micali and Vazirani for nonbipartite graphs [Micali and Vazirani 80] in place of the maximal matching algorithm for finding a weakly stable matching above. This improves on the best known running time of $O\left(\mathrm{~m}^{2}\right)$ for general SR [Scott 05]. 
Lemmas 1.2 and 1.3 indicate that SR-GRP can be "more tractable" than SR. However, the possible nonexistence of a strongly stable matching motivates the search for weakly stable matchings with desirable properties. A rank-maximal matching [Irving et al. 06, Kavitha and Shah 06] includes the maximum possible number of rank-1 edges, and subject to this, the maximum possible number of rank-2 edges, and so on. More formally, define the signature of a matching $M$ as $\left\langle s_{1}, s_{2}, \ldots, s_{m}\right\rangle$, where $s_{i}$ is the number of rank- $i$ edges in $M$. Then a matching is rank-maximal if and only if it has the lexicographic-maximal signature among all matchings.

Recall from Lemma 1.3 that a strongly stable matching is perfect on rank-1 edges, and subject to removing the matched vertices, perfect on rank-2 edges, and so on. It is clear that a rank-maximal matching is strongly stable, when strong stability is possible. If no strongly stable matching exists, then a rankmaximal matching, which by Lemma 1.2 is always weakly stable, seems a natural substitute. [Irving et al. 06] gives an $O(\min (n+R, R \sqrt{n}) m)$ algorithm for the problem of finding a rank-maximal matching in a bipartite graph, where $R$ is the rank of the worst-ranked edge in the matching.

Other desirable types of weakly stable matchings may be those that have maximum cardinality, are egalitarian, are of minimum regret, or admit the fewest number of weakly blocking pairs. An egalitarian (respectively minimum-regret) weakly stable matching satisfies the property that the sum of the ranks (respectively the maximum rank) of the edges is minimized, taken over all weakly stable matchings. Given a general SR instance $I$, each of the problems of finding an egalitarian and a minimum-regret weakly stable matching is NP-hard [Feder 92, Manlove et al. 02] (in the former case, even if the preference lists are complete and strictly ordered, and in the latter case, even if the underlying graph is bipartite). However, the complexity of the problem of finding a weakly stable matching with the minimum number of weakly blocking pairs in $I$ has, until now, been open.

\section{I.3. Related Work}

Part of the motivation for this work is the investigation of which problems become more tractable in SR-GRP as compared to SR, and which problems maintain their hardness. Work along these lines has been done before [Bartholdi and Trick 86, Tan 91, Chung 00, Arkin et al. 09]. In particular, in the case of SR instances where preference lists may include ties, [Chung 00] shows that the "no odd ring" condition on preferences is sufficient for the existence of a weakly stable matching. The SR-GAP acyclic condition is a restriction of the "no odd ring" condition, in that neither odd nor even rings are permitted. 
As previously mentioned, several recent papers have focused on instances of SR-GAP that arise from P2P networks. In particular, [Lebedev et al. 07] independently proved Lemma 1.2 by showing that every instance of SR-GAP (and hence SR-GRP by Proposition 1.1) admits a weakly stable matching. [Gai et al. 07a] showed that every instance of SR with a master list is an instance of SRGAP, but the converse need not be true. The authors also considered instances of SR-SYM, that is, the restriction of SR-GRP where preferences are symmetric. The notion of symmetric preferences is defined formally in Section 3, but informally, an SR instance involves symmetric preferences if for any two agents $u$ and $v, v$ appears in the $k$ th tie in $u$ 's preference list if and only if $u$ appears in the $k$ th tie in $v$ 's preference list. See also [Gai et al. 07b, Mathieu 07a, Mathieu 07b] for further results regarding instances of SR-GAP arising from P2P networks.

[Arkin et al. 09] considers the geometric stable roommates problem, which is a restriction of SR-GRP in which the agents are points in $\mathbb{R}^{d}$, all agents are mutually acceptable, and the ranking function maps a pair of agents to the Euclidean distance between them. In this restricted context, Arkin et al. proved the analogues of Lemmas 1.2 and 1.3. They also provided algorithms for finding egalitarian and super-stable matchings (if they exist).

It is shown in [O'Malley 07] that the problem of determining whether an edge belongs to some weakly stable matching in an instance of SM-SYM (the bipartite restriction of SR-SYM) is NP-complete. He also gave generalized algorithms for finding a strongly stable and/or super-stable matching in the capacitated version of SM-SYM, where agents on one side of the bipartition can be allocated to more than one agent on the other side of the bipartition.

[Sng 08] studied popular matchings in the context of SM-SYM. A matching $M$ is popular if there is no other matching $M^{\prime}$ such that the number of agents who prefer their partner in $M^{\prime}$ to $M$ exceeds the number of agents who prefer their partner in $M$ to $M^{\prime}$. Using properties of this preference class, Sng derived a characterization of popular matchings and a polynomial-time algorithm to determine whether a given matching is popular.

Finally, [Ackermann et al. 08] studied two-sided stable marriage markets in which there is no central authority to find a stable matching. Agents in the market propose pairings to other agents, who may reject or tentatively accept the pairing. Ackermann et al. showed that the number of proposals required before the market reaches a stable matching (called the convergence time) can be exponential in the number of agents, even if the agents make locally optimal proposals. As a counterpart to this result, they showed that for markets with correlated preferences, which corresponds to the SR-GRP model, the convergence time is polynomial. 


\section{I.4. Our Contribution}

In Section 2 we present an $O(\min (n+R, R \sqrt{n}) m)$ algorithm for finding a rankmaximal matching given an instance of SR-GRP, where $R$ is the rank of the worstranked edge in the matching. Such a matching is weakly stable, as previously observed. This is the first generalization of the algorithm of [Irving et al. 06] to a nonbipartite setting. In Section 3, we report on hardness results for each of the problems of finding weakly stable matchings that are of maximum size, are egalitarian, have minimum regret, and admit the minimum number of weakly blocking pairs, given an instance of SR-GRP. We also prove that this last problem is inapproximable within a factor of $n^{1-\varepsilon}$, for any $\varepsilon>0$, unless $\mathrm{P}=\mathrm{NP}$. These hardness results apply even in a restricted version of SR-GRP in which the graph $G$ is bipartite, and (in the first three cases) if an agent $v$ is incident to an edge of rank $k$, then $v$ is incident to an edge of rank $k^{\prime}$, for $1 \leq k^{\prime} \leq k$.

\section{Rank-Maximal Matching}

Consider an instance of SR-GRP represented by an undirected graph $G=(V, E)$ and a ranking function, rank, on $E$. One obvious way to construct a rankmaximal matching in $G$ is to find a maximum-weight matching using edge weights that increase exponentially with improving rank. However, with $K$ distinct rank values, Gabow and Tarjan's matching algorithm [Gabow and Tarjan 91] takes $O\left(K^{2} \sqrt{n \alpha(m, n) \lg n} m \lg n\right)$ time, ${ }^{1}$ where $\alpha$ is the inverse Ackermann function. As in the bipartite restriction [Irving et al. 06], our combinatorial algorithm avoids the problem of exponential-sized edge weights, leading to an improved runtime of $O(\min \{n+R, R \sqrt{n}\} m)$, where $R \leq K$ is the rank of the worstranked edge in the matching. In the remainder of this section, we describe our generalization of the algorithm due to [Irving et al. 06]. We encourage the reader to review this paper, which contains an excellent exposition of the overall approach.

Let $G_{i}=\left(V, E_{\leq i}\right)$. Our algorithm begins by constructing a maximum match$\operatorname{ing} M_{1}$ on $G_{1}$. Note that $M_{1}$ is rank-maximal on $G_{1}$ by definition. Then inductively, given a rank-maximal matching $M_{i-1}$ on $G_{i-1}$, the algorithm exhaustively augments $M_{i-1}$ with edges from $E_{i}$ to construct a rank-maximal matching $M_{i}$ on $G_{i}$. One danger in this approach is that an augmenting path may, for example, add two rank- 2 edges and remove one rank-1 edge. This would destroy the rank-maximal matching on rank-1 edges, because the matching would now have one rank-1 edge fewer. In order to ensure rank-maximality, certain types of edges

\footnotetext{
${ }^{1}$ See [Mehlhorn and Michail 05] for an explanation of the $K^{2}$ factor.
} 
are deleted before augmenting. With these edges deleted, it becomes possible to augment $M_{i-1}$ arbitrarily, while still guaranteeing rank-maximality. Hence, we can perform the augmentations using Micali and Vazirani's fast maximum matching algorithm [Micali and Vazirani 80].

In order to generalize the bipartite algorithm in [Irving et al. 06], we perform one additional type of edge deletion in nonbipartite settings. Additionally, we perform a new type of operation that shrinks certain components of the graph into supervertices. Note that this shrinking is separate from any blossom shrinking [Edmonds 65] that might occur in the maximum matching subroutine.

To understand the edge deletions and component shrinking, we review the Gallai-Edmonds decomposition technique [Lovász and Plummer 86]. This states that $V$ can be partitioned into three sets: GED-U $[G]$, GED-O $[G]$, and GED-P $[G]$. Vertices in GED-U $[G]$ are underdemanded, since they are unmatched in some maximum matching of $G$. All other vertices that are adjacent to one in GED-U $[G]$ are overdemanded and belong to GED-O $[G]$. Finally, all remaining vertices are perfectly demanded and belong to GED-P $[G]$. The decomposition lemma gives many useful structural properties of maximum matchings. For example, in every maximum matching, vertices in GED-O $[G]$ are always matched, and their partner is in GED-U $[G]$. Similarly, vertices in GED-P $[G]$ are always matched, though their partners are also in GED-P $[G]$. We will use the properties given in Lemma 2.1. Note that in the following lemma, and through the rest of the exposition, the cardinality of a connected component $C$ of a graph is the number of vertices in $C$.

Lemma 2.I. (Gallai-Edmonds decomposition.) In any maximum matching $M$ of $G$,

(1) for all $u$ in GED-O $[G], M(u)$ is in GED-U $[G]$;

(2) for all even (cardinality) components $C$ of $G \backslash$ GED-O $[G]$, (i) $C \subseteq$ GED-P $[G]$ and (ii) $M(u)$ is in $C$, for all $u$ in $C$;

(3) for all odd (cardinality) components $C$ of $G \backslash$ GED-O $[G]$, (i) $C \subseteq$ GED-U $[G]$, (ii) $M(u)$ is in $C$, for all $u$ in $C$ except one, say $v$, and (iii) either $v$ is unmatched in $M$, or $M(v)$ is in GED-O $[G]$.

Consider the first inductive step of the algorithm, in which we are trying to construct a rank-maximal matching $M_{2}$ of $G_{2}=\left(V, E_{\leq 2}\right)$, given a maximum matching $M_{1}$ of $G_{1}=\left(V, E_{1}\right)$. We do not want to commit to edges in $M_{1}$ at this point, because perhaps no rank-maximal matching on $G_{2}$ contains these edges. However, according to different parts of the decomposition lemma above, we can safely delete any edge $e=\{u, v\}$ such that either 

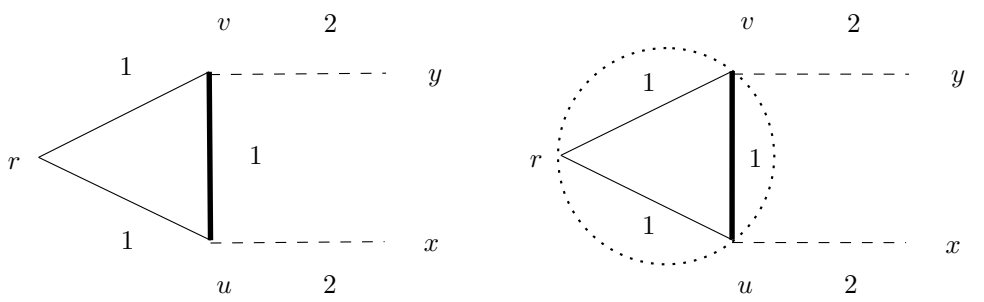

Figure I. Example of a shrinking operation.

(i) $u \in$ GED-O $\left[G_{1}\right]$ and $v \in$ GED-O $\left[G_{1}\right] \cup$ GED-P $\left[G_{1}\right]$ (by part 1 ), or

(ii) $e \in E_{\geq 2}$, and $u \in$ GED-O $\left[G_{1}\right] \cup$ GED-P $\left[G_{1}\right]$ (by parts 1 and 2), or

(iii) $e \in E_{\geq 2}$, and both $u$ and $v$ belong to the same odd component of $G_{1}$ (by part 3).

We delete all such edges to ensure they are not subsequently added to the matching when we augment. Note that the third deletion type is required for nonbipartite graphs, since only one vertex in each odd component $C$ is unmatched internally.

After deleting edges in $G_{1}$, we shrink each odd component $C$ into a supervertex. We define the root $r$ of $C$ as the one vertex in $C$ that is unmatched within $C$. Note that $C$ 's supervertex is matched if and only if $r$ is matched. Now when we add undeleted edges from $e=\{u, v\} \in E_{\geq 2}$ into the graph, if $u \in C$ and $v \notin C$, we replace $e$ with an edge between $v$ and $C$ 's supervertex. Note that during the course of the algorithm, we will be dealing with graphs containing supervertices, which themselves recursively contain supervertices. In such graphs, we define a legal matching to be any collection of independent edges such that in every supervertex, all top-level vertices but the root are matched internally.

To give some intuition for why we shrink odd components, consider the graph in Figure 1. The triangle of rank-1 edges is an odd component $O$ (with $\{u, v\}$ matched), and so neither rank-2 edge is deleted. One way to augment this graph is to include the two rank- 2 edges and take out the rank-1 $\{u, v\}$ edge. This destroys the rank-maximal matching on $G_{1}$. If we shrink the triangle down to a supervertex $O$, however, then $O$ is unmatched, and so $\{O, x\}$ and $\{O, y\}$ are both valid augmenting paths. Note how these augmenting paths can be expanded inside the supervertex $O$ by removing and adding one rank-1 edge to end at the root $r$. This expansion makes the augmenting path legal in the original graph, while not changing the number of matching edges internal to the supervertex.

Algorithm 1 contains pseudocode for our nonbipartite rank-maximal matching algorithm. One aspect that requires more explanation is how we augment $M_{i}$ in 


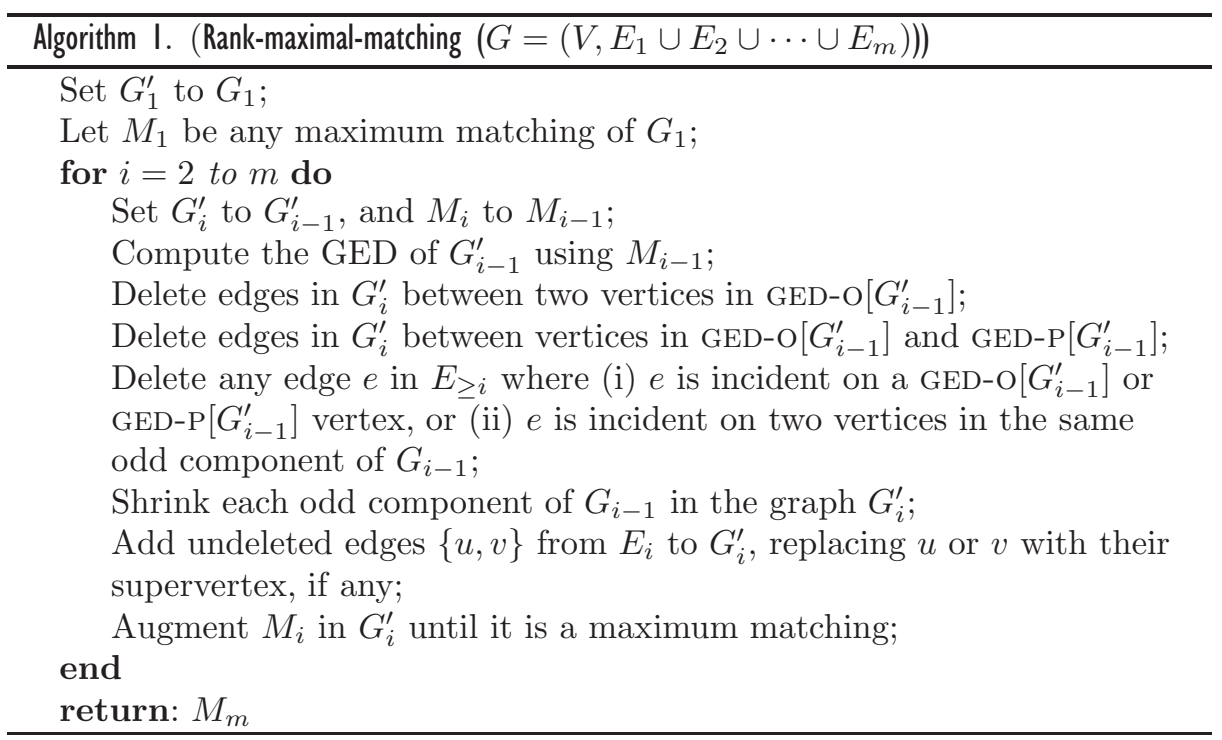

$G_{i}^{\prime}$. The overall approach is to find an augmenting path $P$ while regarding each top-level supervertex in $G_{i}^{\prime}$ as a regular vertex. Then for each supervertex $C$ in $P$, we expand $P$ through $C$ in the following way. Let $u$ be the vertex in $C$ that $P$ enters along an unmatched edge. If $u$ is the root $r$ of $C$, then $C$ is unmatched, and we can replace $C$ by $u$ in $P$. Otherwise, $u \neq r$, and either $C$ is unmatched or $P$ leaves $C$ via the matched edge incident on $r$. In the next lemma, we show that there is an even-length alternating path from $u$ to $r$, beginning with a matched edge. We can expand $P$ by replacing $C$ with this even-length alternating path.

Lemma 2.2. Let $M$ be a legal matching on some supervertex $C$ with root $r$. Let $u$ be any other node in $C$. Then there is an even-length alternating path from u to $r$ beginning with a matched edge.

Proof. Let $M^{\prime}$ be a legal matching of $C$ in which $u$ is unmatched (such a matching is guaranteed by the decomposition lemma). Consider the symmetric difference of $M$ and $M^{\prime}$. Since every vertex besides $u$ and $r$ is matched in both matchings, there must be an even-length alternating path consisting of $M$ and $M^{\prime}$ edges from $u$ to $r$.

In all cases of $P$ and $C$, note that $C$ has the same number of internally matched edges before and after augmentation by $P$, and so the matching remains legal. Also, if $r$ was matched prior to augmentation, then it is still matched afterward. 
The next three lemmas, which generalize those in [Irving et al. 06], establish the correctness of the algorithm. Lemma 2.3 proves that no rank-maximal matching contains a deleted edge. Lemma 2.4 proves that augmenting a rank-maximal matching $M_{i-1}$ of $G_{i-1}$ does not change its signature up to rank $(i-1)$. And finally, Lemma 2.5 proves that the final matching is rank-maximal on the original graph $G$.

Lemma 2.3. Suppose that every rank-maximal matching of $G_{i-1}$ is a maximum legal matching of $G_{i-1}^{\prime}$. Then every rank-maximal matching of $G_{i}$ is contained in $G_{i}^{\prime}$.

Proof. Let $M$ be an arbitrary rank-maximal matching of $G_{i}$. Then $M \cap E_{\leq i-1}$ is a rank-maximal matching of $G_{i-1}$, and by assumption, a maximum legal matching of $G_{i-1}^{\prime}$. By Lemma 2.1, the edges we delete when constructing $G_{i}^{\prime}$ belong to no maximum matching of $G_{i-1}^{\prime}$; in particular, $M \cap E_{\leq i-1}$. Now, since $M=\left(M \cap E_{\leq i-1}\right) \cup\left(M \cap E_{i}\right)$, it remains to show that $M \cap E_{i}$ contains no deleted edges.

Suppose for the sake of a contradiction that there is an edge $e \in M \cap E_{i}$ that is deleted by the algorithm. Note that $e \in E_{i}$ is deleted only if $e$ is incident on (i) a GED-O $\left[G_{i-1}^{\prime}\right]$ vertex, (ii) a GED-P $\left[G_{i-1}^{\prime}\right]$ vertex, or (iii) two vertices in the same odd component of $G_{i-1}^{\prime}$. In each of these cases, $e \in M \cap E_{i}$ means that $M \cap E_{\leq i-1}$ cannot be a rank-maximal matching of $G_{i-1}$, giving the required contradiction.

Lemma 2.4. Let $M_{i}$ and $M_{j}$ be the matchings produced by the algorithm, where $i<j$. Then $M_{i}$ and $M_{j}$ have the same number of edges with rank at most $i$.

Proof. $M_{i}$ consists of edges contained within top-level supervertices of $G_{i}^{\prime}$, and edges between top-level (super)vertices of $G_{i}^{\prime}$. We have already shown that augmenting through a supervertex does not change the number of matching edges internal to the supervertex. Hence $M_{j}$ contains the same number of such edges as $M_{i}$.

By Lemma 2.1, the remaining edges of $M_{i}$ are all incident on some GED-O $\left[G_{i}^{\prime}\right]$ or GED-P $\left[G_{i}^{\prime}\right]$ (super)vertex. Since these vertices are matched in $M_{i}$, they are also matched in $M_{j}$, since augmenting does not affect the matched status of a vertex. Also, no edges of rank worse than $i$ are incident on such vertices, due to deletions, and so each must be matched along a rank- $i$ edge or better in $M_{j}$. Hence $\left|M_{i}\right| \leq\left|M_{j} \cap E_{\leq i}\right|$. Of course, $\left|M_{j} \cap E_{\leq i}\right| \leq\left|M_{i}\right|$, since all edges from $E_{\leq i}$ in $G_{j}^{\prime}$ are also in $G_{i}^{\prime}$, and $M_{i}$ is a maximum legal matching of $G_{i}^{\prime}$. 
Lemma 2.5. For every $i$, the following statements hold:

(1) Every rank-maximal matching of $G_{i}$ is a maximum legal matching of $G_{i}^{\prime}$.

(2) $M_{i}$ is a rank-maximal matching of $G_{i}$.

Proof. For the base case, rank-maximal matchings are maximum matchings on rank-1 edges, and so both statements hold for $i=1$. By Lemma 2.3 and the inductive hypothesis, every rank-maximal matching of $G_{i}$ is contained in $G_{i}^{\prime}$. Let $\left\langle s_{1}, s_{2}, \ldots, s_{i}\right\rangle$ be the signature of such a matching. By Lemma 2.4, $M_{i}$ has the same signature as $M_{i-1}$ up to rank $(i-1)$. Hence $M_{i}$ 's signature is $\left\langle s_{1}, s_{2}, \ldots, s_{i-1}, t_{i}\right\rangle$ for some $t_{i} \leq s_{i}$, since $M_{i-1}$ is a rank-maximal matching of $G_{i-1}$. However, $M_{i}$ is a maximum legal matching of $G_{i}^{\prime}$, and hence $t_{i}=s_{i}$ and $M_{i}$ is a rank-maximal matching of $G_{i}$. This proves the second statement.

For the first statement, let $N_{i}$ be any rank-maximal matching of $G_{i}$. By Lemma 2.3 and the inductive hypothesis, we know that $N_{i}$ is contained in $G_{i}^{\prime}$. Furthermore, $N_{i}$ has signature $\left\langle s_{1}, s_{2}, \ldots, s_{i}\right\rangle$, which is the same signature as that of $M_{i}$. Hence $N_{i}$ is also a maximum legal matching of $G_{i}^{\prime}$.

We now comment on the runtime of the algorithm. In each iteration $i$, it is clear that computing the decomposition (given a maximum matching), deleting edges, and shrinking components all take $O(m)$ time. Constructing $M_{i}$ from $M_{i-1}$ requires $\left|M_{i}\right|-\left|M_{i-1}\right|+1$ augmentations. At the top level of augmenting (when supervertices are regarded as vertices), we can use the Micali-Vazirani nonbipartite matching algorithm, which runs in time $O\left(\min \left(\sqrt{n},\left|M_{i+1}\right|-\left|M_{i}\right|+1\right) m\right)$.

Next, we have to expand each augmenting path $P$ through its incident supervertices. Let $u$ be the first vertex of some supervertex $C$ that $P$ enters along an unmatched edge. It is clear that we can do this expansion in time linear in the size of $C$ by appending a dummy unmatched vertex $d$ to $u$, and then looking for an augmenting path from $d$ to $r$ in $C$. Since each supervertex belongs to at most one augmenting path in each round of the Micali-Vazirani algorithm, this does not affect the asymptotic runtime.

It follows that after $R$ iterations, the runtime is at most $O(\min (n+R, R \sqrt{n}) m)$. Using the idea in [Irving et al. 06], we can stop once $R$ is the rank of the worstranked edge in a rank-maximal matching, because we can test in $O(m)$ time whether $M_{R}$ is a maximum matching of $G_{R}$ together with all undeleted edges of rank worse than $R$ (in which case $M_{R}$ is rank-maximal).

Theorem 2.6. Suppose we are given an instance of SR-GRP represented by an undirected graph $G=(V, E)$ and a ranking function rank on $E$. Let $R$ be the rank of the worst-ranked edge in a rank-maximal matching of $G=(V, E)$. Then a rank-maximal matching of $G$ can be found in time $O(\min (n+R, R \sqrt{n}) m)$. 


\section{Hardness Results}

In this section we establish several NP-hardness results for a special case of SR-GRP. We refer to this restriction as STABLE MARRIAGE WITH SYMMETRIC PREFERENCES (SM-SYM). An instance of SM-SYM is an instance of SR in which the underlying graph is bipartite (with men and women representing the two sets of agents in the bipartition), subject to the restriction that a woman $w_{j}$ appears in the $k$ th tie in a man $m_{i}$ 's preference list if and only if $m_{i}$ appears in the $k$ th tie in $w_{j}$ 's preference list. Clearly, an instance of SM-SYM is a bipartite instance of SR-GRP in which $\operatorname{rank}\left(\left\{m_{i}, w_{j}\right\}\right)=k$ if and only if $w_{j}$ appears in the $k$ th tie in $m_{i}$ 's preference list for every man $m_{i}$ and woman $w_{j}$. Indeed, it will be helpful to assume subsequently that rank is defined implicitly in this way, given an instance of SM-SYM.

Our first result demonstrates the NP-completeness of COM-SM-SYM, which is the problem of deciding whether a complete weakly stable matching (i.e., a weakly stable matching in which everyone is matched) exists given an instance of SM-SYM. Our transformation begins from EXACT-MM, which is the problem of deciding, given a graph $G$ and an integer $K$, whether $G$ admits a maximal matching of size $K$.

Theorem 3.I. COM-SM-SYM is NP-complete.

Proof. Clearly COM-SM-SYM is in NP. To show NP-hardness, we reduce from EXACT-MM in subdivision graphs, which is NP-complete [Manlove and O'Malley 05]. Let $G=(V, E)$, a subdivision graph of some graph $G^{\prime}$, and let $K$, a positive integer, be an instance of EXACT-MM. Suppose that $V=U \cup W$ is a bipartition of $G$, where $U=\left\{m_{1}, m_{2}, \ldots, m_{n_{1}}\right\}$ and $W=\left\{w_{1}, w_{2}, \ldots, w_{n_{2}}\right\}$. Then we denote the set of vertices adjacent to a vertex $m_{i} \in U$ in $G$ by $W_{i}$ and similarly the set of vertices adjacent to $w_{j} \in W$ in $G$ by $U_{j}$.

We construct an instance $I$ of COM-SM-SYM as follows: Let $U \cup X \cup A \cup B$ be the set of men and $W \cup Y \cup A^{\prime} \cup B^{\prime}$ the set of women, where $X=\left\{x_{1}, x_{2}, \ldots, x_{n_{2}-K}\right\}$, $Y=\left\{y_{1}, y_{2}, \ldots, y_{n_{1}-K}\right\}, A=\left\{a_{1}, a_{2}, \ldots, a_{K}\right\}, B=\left\{b_{1}, b_{2}, \ldots, b_{K}\right\}, A^{\prime}=$ $\left\{a_{1}^{\prime}, a_{2}^{\prime}, \ldots, a_{K}^{\prime}\right\}$ and $B^{\prime}=\left\{b_{1}^{\prime}, b_{2}^{\prime}, \ldots, b_{K}^{\prime}\right\}$. The preference lists of $I$ are shown in Table 1 (entries in parentheses are tied). It may be verified that $I$ is an instance of SM-SYM (e.g., for any $x_{i} \in X$ and for any $w_{j} \in W$, each of $x_{i}$ and $w_{j}$ appears in the second tie in each other's list). We claim that $G$ has an exact maximal matching of size $K$ if and only if $I$ admits a complete weakly stable matching.

Suppose $G$ has a maximal matching $M$, where $|M|=K$. We construct a matching $M^{\prime}$ in $I$ as follows. Initially let $M^{\prime}=M$. There remain $n_{1}-K$ men in $U$ that are not assigned to women in $W$; denote these men by $m_{k_{i}}$ 


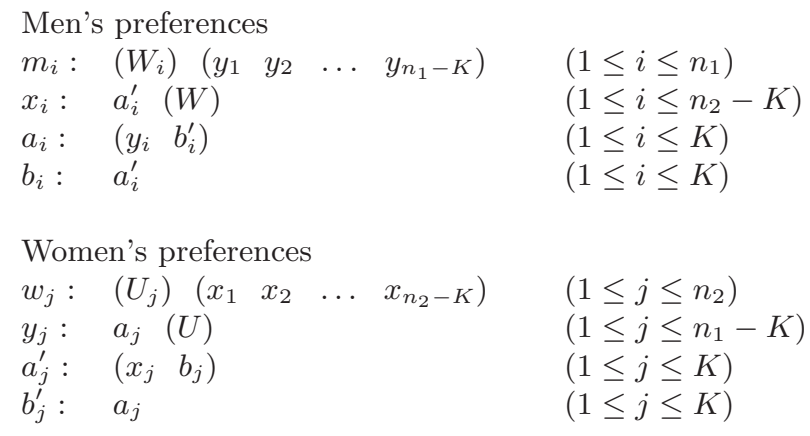

Table I. Preference lists for the constructed instance of COM-SM-SYM.

$\left(1 \leq i \leq n_{1}-K\right)$ and add $\left(m_{k_{i}}, y_{i}\right)$ to $M^{\prime}$. Similarly, there remain $n_{2}-K$ women in $W$ that are not assigned to men in $U$; denote these women by $w_{l_{j}}$ $\left(1 \leq j \leq n_{2}-K\right)$, and add $\left(x_{j}, w_{l_{j}}\right)$ to $M^{\prime}$. Finally, we add $\left(a_{i}, b_{i}^{\prime}\right)$ and $\left(b_{i}, a_{i}^{\prime}\right)$, $1 \leq i \leq K$, to $M^{\prime}$. It may then be verified that $M^{\prime}$ is a complete weakly stable matching in $I$.

Conversely, suppose that $M^{\prime}$ is a complete weakly stable matching in $I$. Let $M=M^{\prime} \cap E$. We now show that $|M|=K$. First suppose that $|M|<K$. Then since $M^{\prime}$ is a complete weakly stable matching, at least $n_{1}-K+1$ men in $U$ must be assigned in $M^{\prime}$ to women in $Y$, which is impossible, since there are only $n_{1}-K$ women in $Y$. Now suppose $|M|>K$. Hence at most $n_{1}-K-1$ women in $Y$ are assigned in $M^{\prime}$ to men in $U$. Then since $M^{\prime}$ is complete, there exists at least one woman in $Y$ assigned in $M^{\prime}$ to a man in $A$. Thus at most $K-1$ men in $A$ are assigned in $M^{\prime}$ to women in $B^{\prime}$. Hence only $K-1$ women in $B^{\prime}$ are assigned in $M^{\prime}$, contradicting the fact that $M^{\prime}$ is a compete weakly stable matching in $I$. Finally, it is straightforward to verify that $M$ is maximal in $G$.

The following corollary will be useful for establishing subsequent results.

Corollary 3.2. COM-SM-SYM is NP-complete, even if each preference list comprises exactly two ties (where a tie can be of length 1).

Proof. The constructed instance in the proof of Theorem 3.1 can be extended to that shown in Table 2 with straightforward modifications to the proof of correctness. This allows us to assume that each man has exactly two ties on his list, and each woman has exactly two ties on hers.

We next consider minimum-regret and egalitarian weakly stable matchings, given an instance $I$ of SMC-SYM, which is the restriction of SM-SYM in which 


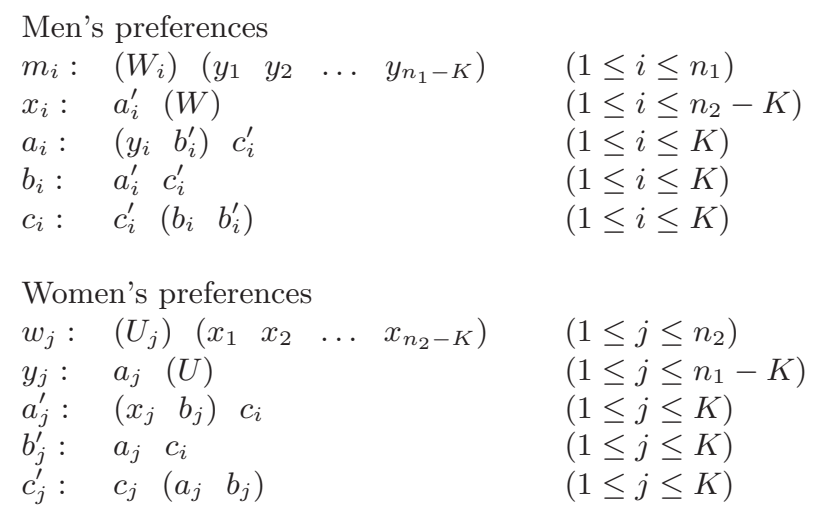

Table 2. Preference lists for the extended constructed instance of COM-SM-SYM.

each person finds all members of the opposite sex acceptable. Let $U$ and $W$ be the sets of men and of women in $I$ respectively, let $M$ be a weakly stable matching in $I$, and let $p$ be some agent in $I$. Then we define the cost of $p$ with respect to $M$, denoted by $\operatorname{cost}_{M}(p)$, to be $\operatorname{rank}(\{p, M(p)\})$. Furthermore, we define the regret of $M$, denoted by $r(M)$, to be $\max _{p \in U \cup W} \operatorname{cost}_{M}(p)$. We say that $M$ has minimum regret if $r(M)$ is minimized over all weakly stable matchings in $I$. Similarly, we define the cost of $M$, denoted by $c(M)$, to be $\sum_{p \in U \cup W} \operatorname{cost}_{M}(p)$. Then $M$ is said to be egalitarian if $c(M)$ is minimized over all weakly stable matchings in $I$.

We define REGRET-SMC-SYM (respectively EGAL-SMC-SYM) to be the problem of deciding, given an instance $I$ of SMC-SYM and a positive integer $K$, whether $I$ admits a weakly stable matching such that $r(M) \leq K$ (respectively $c(M) \leq K$ ). We now show that REGRET-SMC-SYM is NP-complete.

Theorem 3.3. REGRET-SMC-SYM is NP-complete.

Proof. Clearly the problem belongs to NP. To show NP-hardness, we reduce from the restriction of COM-SM-SYM in which each person's list has exactly two ties, which is NP-complete by Corollary 3.2. Let $I$ be such an instance of this problem. We form an instance $I^{\prime}$ of REGRET-SMC-SYM as follows. Initially, the people and preference lists in $I$ and $I^{\prime}$ are identical. Next, in $I^{\prime}$, each person adds a third tie in his or her preference list containing all members of the opposite sex that are not already contained in the first two ties. It is not difficult to verify that $I$ admits a complete weakly stable matching if and only if $I^{\prime}$ admits a weakly stable matching $M$ such that $r(M) \leq 2$. 


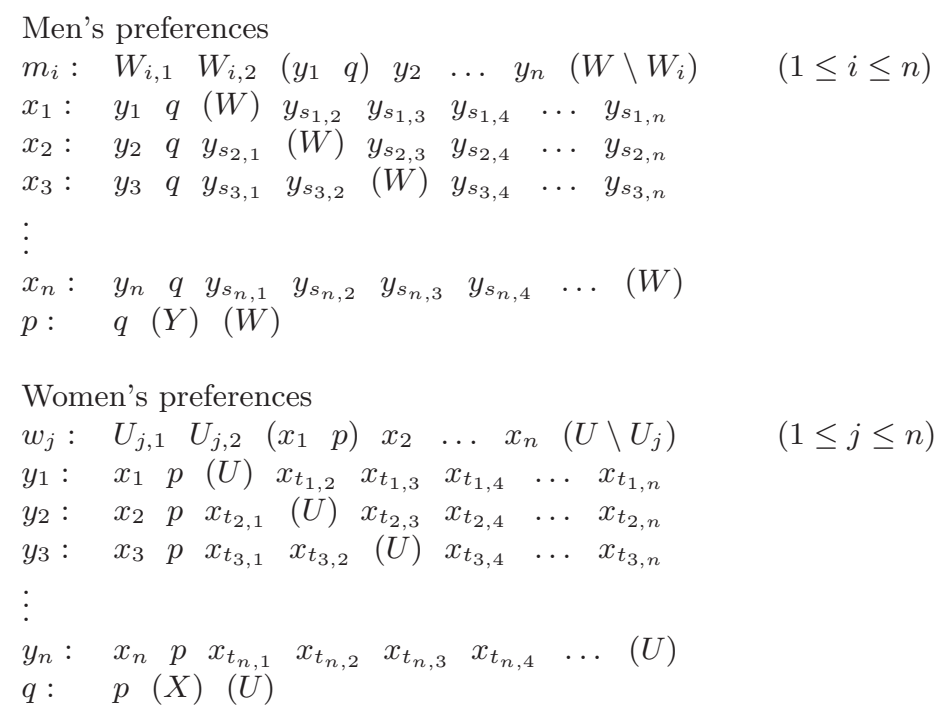

Table 3. Preference lists for the constructed instance of EGAL-SMC-SYM.

We next prove that EGAL-SMC-SYM is NP-complete, using a result from [Gergely 74], shown in Theorem 3.4, relating to diagonalized Latin squares. A transversal of an order- $n$ Latin square is a set $S$ of $n$ distinct-valued cells, no two of which are in the same row or column. A Latin square is said to be diagonalized if the main diagonal is a transversal.

Theorem 3.4. [Gergely 74] For any integer $n \geq 3$, there exists a diagonalized Latin square of order $n$ having a transversal that has no common entry with the main diagonal.

Theorem 3.5. EGAL-SMC-SYM is NP-complete.

Proof. Clearly EGAL-SMC-SYM is in NP. To show NP-hardness, we reduce from the restriction of COM-SM-SYM in which each person's list has exactly two ties, which is NP-complete by Corollary 3.2. Let $I$ be such an instance of this problem, where $U=\left\{m_{1}, m_{2}, \ldots, m_{n}\right\}$ is the set of men and $W=\left\{w_{1}, w_{2}, \ldots, w_{n}\right\}$ is the set of women. For each man $m_{i} \in U(1 \leq i \leq n)$ we denote the women in the first and second ties on $m_{i}$ 's preference list in $I$ by $W_{i, 1}$ and $W_{i, 2}$ respectively, and let $W_{i}=W_{i, 1} \cup W_{i, 2}$. Similarly, for each woman $w_{j} \in W(1 \leq j \leq n)$ we denote the men in the first and second ties on $w_{j}$ 's preference list in $I$ by $U_{j, 1}$ and $U_{j, 2}$ respectively, and let $U_{j}=U_{j, 1} \cup U_{j, 2}$. 
We construct an instance $I^{\prime}$ of EGAL-SMC-SYM as follows: Let $U \cup X \cup\{p\}$ be the set of men and let $W \cup Y \cup\{q\}$ be the set of women, where $X=\left\{x_{1}, x_{2}, \ldots, x_{n}\right\}$ and $Y=\left\{y_{1}, y_{2}, \ldots, y_{n}\right\}$. Then we construct the preference lists in $I^{\prime}$ by considering the diagonalized Latin square $S=\left(s_{i, j}\right)$ of order $n$, as constructed using Gergely's method [Gergely 74] (we note that Gergely's method is polynomialtime computable). Without loss of generality we may assume that the entries in the main diagonal are in the order $1,2, \ldots, n$ (this can be achieved by simply permuting symbols in $S$ if necessary). Next we construct a matrix $T=\left(t_{i, j}\right)$ from $S$ as follows: for each $i$ and $j(1 \leq i, j \leq n)$, if $s_{i, j}=k$ then $t_{k, j}=i$. We claim that $T$ is a Latin square.

For suppose not. First suppose $t_{i, j}=t_{i, k}=l$, for some $j \neq k$. Thus it follows that $s_{l, j}=s_{l, k}=i$, contradicting the fact that $S$ is a Latin square. Now suppose $t_{i, j}=t_{k, j}=l$, for some $i \neq k$. Therefore $s_{l, j}=i$ and $s_{l, j}=k$, which is impossible. Hence $T$ is a Latin square. Moreover, the elements $1,2, \ldots, n$ appear in order on the main diagonal of $T$.

We then use $S$ and $T$ to construct the preference lists as shown in Table 3 . By the construction of $T$ from $S$ and by inspection of the remaining preference list entries, we observe that $I^{\prime}$ is an instance of EGAL-SMC-SYM. Intuitively, we require the Latin squares $S$ and $T$ in this construction to ensure that the preference lists remain symmetric after including the agents in $Y$ and $X$ in the preference lists of the agents in $U$ and $W$ respectively. For example, each $y_{2}$ appears in the fourth tie in the preference list of each $m_{i} \in U$, so all men in $U$ must appear in the fourth tie in $y_{2}$ 's list. Thus the first, second, and third ties in $y_{2}$ 's list must be nonempty. In particular, $x_{t_{2,1}}$ appears in the third tie in $y_{2}$ 's list. Let $t_{2,1}=k$. Then by construction, $s_{k, 1}=2$, and it may be verified that $y_{s_{k, 1}}=y_{2}$ appears in the third tie in $x_{k}$ 's list, since $k>1$.

Let $K=2(3 n+1)$. We now show that $I$ has a complete weakly stable matching $M$ if and only if $I^{\prime}$ has a weakly stable matching $M^{\prime}$ such that $c\left(M^{\prime}\right) \leq K$.

Suppose that $M$ is a complete weakly stable matching in $I$. Then we construct a matching $M^{\prime}$ in $I^{\prime}$ as follows: $M^{\prime}=M \cup\{(p, q)\} \cup\left\{\left(x_{i}, y_{i}\right): 1 \leq i \leq n\right\}$. Then clearly $M^{\prime}$ is weakly stable in $I^{\prime}$, since $M$ is weakly stable in $I$ and every person in $X \cup Y \cup\{p, q\}$ is assigned to his or her first-choice partner. Also, each person $a_{i} \in U \cup W$ has $\operatorname{cost}_{M^{\prime}}\left(a_{i}\right) \leq 2$, and each person $a_{j} \in X \cup Y \cup\{p, q\}$ has $\operatorname{cost}_{M^{\prime}}\left(a_{j}\right)=1$. Therefore $c\left(M^{\prime}\right) \leq(2 n+n+1)+(2 n+n+1)=K$.

Conversely, suppose that $M^{\prime}$ is a weakly stable matching in $I^{\prime}$ such that $c\left(M^{\prime}\right) \leq K$. We observe that $p$ and $q$ are assigned to each other in every weakly stable matching and also that $x_{i}$ is assigned to $y_{i}(1 \leq i \leq n)$ in every weakly stable matching. Hence each man $m_{i}$ is assigned in $M^{\prime}$ to a woman in $W_{i}$ or a woman in $W \backslash W_{i}$. Now suppose there exists a man $m_{k} \in U$ such that $M^{\prime}\left(m_{k}\right) \in W \backslash W_{k}$. Then $\operatorname{cost}_{M^{\prime}}\left(m_{k}\right)=n+3$, and since the 
preference lists are symmetric, $\operatorname{cost}_{M^{\prime}}\left(M^{\prime}\left(m_{k}\right)\right)=n+3$. Hence $c\left(M^{\prime}\right) \geq$ $((n+3)+(n-1)+n+1)+((n+3)+(n-1)+n+1)=3 n+3+3 n+3=6(n+1)$, contradicting the fact that $c\left(M^{\prime}\right) \leq K$. Thus each man $m_{i} \in U$ and woman $w_{j} \in W$ must be assigned to a partner in $W_{i}$ and $U_{j}$ respectively. Now let $M=M^{\prime} \backslash\left(\{(p, q)\} \cup\left\{\left(x_{i}, y_{i}\right): 1 \leq i \leq n\right\}\right)$. Then since each person $a_{i} \in U \cup W$ is assigned to someone in $W_{i} \cup U_{i}$ in $M$ and since $M^{\prime}$ is weakly stable in $I^{\prime}$, it follows that $M$ is a complete weakly stable matching in $I$.

Our final hardness result applies to SM-GRP, which is the restriction of SR-GRP to bipartite graphs. Recall that a strongly stable matching has no weakly blocking pairs. MIN-BP-SM-GRP is the problem of finding a weakly stable matching (which by definition has no strongly blocking pairs) with the minimum number of weakly blocking pairs, given an instance of SM-GRP.

Theorem 3.6. MIN-BP-SM-GRP is not approximable within a factor of $n^{1-\varepsilon}$, for any $\varepsilon>0$, unless $P=N P$, where $n$ is the number of men and women.

Proof. Let $\varepsilon>0$. We give a gap-introducing reduction from the restriction of COMSM-SYM in which each person's list has exactly two ties, which is NP-complete by Corollary 3.2. Let $I$ be an instance of this problem, where $U=\left\{m_{1}, m_{2}, \ldots, m_{n}\right\}$ is the set of men and $W=\left\{w_{1}, w_{2}, \ldots, w_{n}\right\}$ is the set of women. For each man $m_{i} \in U$, we let $W_{i, 1}$ and $W_{i, 2}$ denote the women in the first and second ties in $m_{i}$ 's preference list in $I$, respectively. Similarly, for each woman $w_{j} \in W$, we let $U_{j, 1}$ and $U_{j, 2}$ denote the men in the first and second ties in $w_{j}$ 's preference list in $I$, respectively.

Construct an instance $I^{\prime}$ of MIN-BP-SM-GRP with $U \cup X$ as the set of men and $W \cup Y$ as the set of women, where $X=\left\{x_{1}, x_{2}, \ldots, x_{n^{2+k}+1}\right\}$ and $Y=$ $\left\{y_{1}, y_{2}, \ldots, y_{n^{2+k}+1}\right\}$, where $k=\left\lceil\frac{4}{\varepsilon}\right\rceil$. The preferences for these people are given in Table 4. It is clear from Table 4 that $I^{\prime}$ is an instance of MIN-BP-SM-GRP, and additionally that $I^{\prime}$ can be constructed in polynomial time.

Suppose $I$ admits a complete weakly stable matching $M$. Augment $M$ in $I^{\prime}$ to form $M^{\prime}$ by pairing all people in $X$ and $Y$. Note that $M^{\prime}$ is weakly stable with at most $n^{2}$ weakly blocking pairs in $I^{\prime}$, since any weakly blocking pair of $M^{\prime}$ must also weakly block $M$ in $I$, and $I$ has at most $n^{2}$ acceptable pairs.

Now suppose that $I$ does not admit a complete weakly stable matching. Let $M^{\prime}$ be a weakly stable matching in $I^{\prime}$. We claim that $M^{\prime}$ admits at least $n^{2+k}+1$ weakly blocking pairs in $I^{\prime}$. For suppose not. Then every man in both $U$ and $X$ must be matched in $M^{\prime}$, for otherwise he weakly blocks with each of the $n^{2+k}+1$ women in $Y$, giving a contradiction. It follows that $M^{\prime}$ must contain a perfect matching from $X$ to $Y$, since (i) every man in $X$ is matched in $M^{\prime}$, 


$$
\begin{aligned}
& \text { Men's preferences } \\
& \text { rank-1 rank-2 rank-3 } \\
& m_{i}: \quad\left(W_{i, 1}\right) \quad\left(W_{i, 2}\right) \quad(Y) \quad(1 \leq i \leq n) \\
& \begin{array}{lll}
x_{i}: & (Y) \quad\left(1 \leq i \leq n^{2+k}+1\right)
\end{array} \\
& w_{j}:\left(U_{j, 1}\right) \quad\left(U_{j, 2}\right) \quad(1 \leq j \leq n) \\
& y_{j}: \quad(U \cup X) \quad\left(1 \leq j \leq n^{2+k}+1\right)
\end{aligned}
$$

Table 4. Preference lists for the constructed instance of MIN-BP-SM-GRP.

(ii) $|X|=|Y|$, and (iii) men in $X$ find only women in $Y$ acceptable. Hence, $M^{\prime}$ contains a perfect matching $M$ from $U$ to $W$, which is clearly weakly stable in $I$, a contradiction. Hence the claim is established.

Thus the existence of an approximation algorithm for MIN-BP-SM-GRP with performance guarantee $n^{k}$ could be used to decide in polynomial time whether $I$ admits a complete weakly stable matching, a contradiction unless $\mathrm{P}=\mathrm{NP}$. Finally, we note that $I^{\prime}$ contains a total of $N$ men and women, where $N=$ $2\left(n+n^{2+k}+1\right)$. It follows that $N \leq 6 n^{2+k}$, and hence $n^{k} \geq 6^{-\frac{k}{2+k}} N^{1-\frac{\varepsilon}{2}}$. Without loss of generality we may assume that $n \geq 3$, so that $N \geq 6^{\frac{k}{2}}$ and hence $6^{-\frac{k}{2+k}} \geq N^{-\frac{\varepsilon}{2}}$. It follows that $n^{k} \geq N^{1-\varepsilon}$, as required.

\section{Conclusion}

In this work, we have introduced a restriction of SR in which preferences are deduced from a global ranking of the roommate pairs. This restriction has the property that weakly stable matchings are guaranteed to exist, a property that does not hold in the general SR problem, even if there are no ties in the preference lists. We derived a polynomial-time algorithm to find a rank-maximal (weakly stable) matching. This is the first nonbipartite generalization of the rank-maximal matching algorithm due to [Irving et al. 06]. Also, we have proved several hardness results in an even more restricted setting for each of the problems of finding weakly stable matchings that are of maximum size, are egalitarian, have minimum regret, and admit the minimum number of weakly blocking pairs. We conclude with the following open problem: it remains to extend the rank-maximal matching algorithm of [Irving et al. 06] to an arbitrary instance of SR (i.e., in which preferences need not be deduced from a global ranking of the roommate pairs).

Acknowledgments. We would like to thank Péter Biró and Utku Ünver for helpful remarks concerning relationships between SR-GRP and SR-GAP. We would also like to thank an anonymous referee for helpful remarks on an earlier version of this paper. The first 
author's research was supported in part by NSF grants IIS-0427858 and CCF-0514922. Part of this work was completed while he was visiting Microsoft Research. The second author's work was completed while he was visiting the Computer Science Department, Carnegie Mellon University. The third author's research was supported by EPSRC grant EP/E011993/1. A preliminary version of this paper appeared in [Abraham et al. 07b].

\section{References}

[Abraham et al. 07a] D. J. Abraham, A. Blum, and T. Sandholm. "Clearing Algorithms for Barter Exchange Markets: Enabling Nationwide Kidney Exchanges." In Proceedings of the 8th ACM Conference on Electronic Commerce, pp. 295-304. New York: ACM Press, 2007.

[Abraham et al. 07b] D. J. Abraham, A. Levavi, D. F. Manlove, and G. O'Malley. "The Stable Roommates Problem with Globally-Ranked Pairs." In Internet and Network Economics: Third International Workshop, WINE 2007, San Diego, CA, USA, December 12-14, 2007, Proceedings, Lecture Notes in Computer Science 4858, pp. 431-444. New York: Springer, 2007.

[Ackermann et al. 08] H. Ackermann, P. W. Goldberg, V. S. Mirrokni, H. Röglin, and B. Vöcking. "Uncoordinated Two-Sided Matching Markets." In Proceedings of the 9th ACM Conference on Electronic Commerce, pp. 256-263. New York: ACM, 2008.

[Arkin et al. 09] E. M. Arkin, S. W. Bae, A. Efrat, J. S. B. Mitchell, K. Okamoto, and V. Polishchuk. "Geometric Stable Roommates." Information Processing Letters 109 (2009), 219-224.

[Bartholdi and Trick 86] J. J. Bartholdi and M. A. Trick. "Stable Matchings with Preferences Derived from a Psychological Model." Operations Research Letters 5 (1986), 165-169.

[Cechlárová and Fleiner 05] K. Cechlárová and T. Fleiner. "On a Generalization of the Stable Roommates Problem." ACM Transactions on Algorithms 1:1 (2005), 143-156.

[Chung 00] K. S. Chung. "On the Existence of Stable Roommate Matchings." Games and Economic Behavior 33:2 (2000), 206-230.

[Delmonico 04] F. L. Delmonico. "Exchanging Kidneys-Advances in Living-Donor Transplantation." New England Journal of Medicine 350 (2004), 1812-1814.

[Edmonds 65] J. Edmonds. "Paths, Trees, and Flowers." Canadian Journal of Mathematics 17 (1965), 449-467.

[Feder 92] T. Feder. "A New Fixed Point Approach for Stable Networks and Stable Marriages." Journal of Computer and System Sciences 45 (1992), 233-284.

[Gabow and Tarjan 91] H. N. Gabow and R. E. Tarjan. "Faster Scaling Algorithms for General Graph Matching Problems." Journal of the ACM 38:4 (1991), 815-853.

[Gai et al. 07a] A.-T. Gai, D. Lebedev, F. Mathieu, F. de Montgolfier, J. Reynier, and L. Viennot. "Acyclic Preference Systems in P2P Networks." In Euro-Par 2007 Parallel Processing: 13th International Euro-Par Conference, Rennes, France, August 
28-31, 2007, Proceedings, Lecture Notes in Computer Science 4641, pp. 825-834. New York: Springer, 2007.

[Gai et al. 07b] A.-T. Gai, F. Mathieu, F. de Montgolfier, and J. Reynier. "Stratification in P2P Networks: Application to BitTorrent." In Proceedings of the 27th International Conference on Distributed Computing Systems, ICDCS 2007, p. 30. Washington, DC: IEEE Computer Society, 2007.

[Gale and Shapley 62] D. Gale and L. S. Shapley. "College Admissions and the Stability of Marriage." American Mathematical Monthly 69 (1962), 9-15.

[Gergely 74] E. Gergely. "A Simple Method for Constructing Doubly Diagonalized Latin Squares." Journal of Combinatorial Theory, Series A 16:2 (1974), 266-272.

[Gjertson and Cecka 00] D. W. Gjertson and J. M. Cecka. "Living Unrelated Donor Kidney Transplantation." Kidney International 58 (2000), 491-499.

[Gusfield and Irving 89] D. Gusfield and R. W. Irving. The Stable Marriage Problem: Structure and Algorithms. Cambridge, MA: MIT Press, 1989.

[Irving 85] R. W. Irving. "An Efficient Algorithm for the 'Stable Roommates' Problem." Journal of Algorithms 6 (1985), 577-595.

[Irving and Manlove 02] R. W. Irving and D. F. Manlove. "The Stable Roommates Problem with Ties." Journal of Algorithms 43 (2002), 85-105.

[Irving et al. 06] R. W. Irving, D. Michail, K. Mehlhorn, K. Paluch, and K. Telikepalli. "Rank-Maximal Matchings." ACM Transactions on Algorithms 2:4 (2006), 602-610.

[Irving et al. 08] R. W. Irving, D. F. Manlove, and S. Scott. "The Stable Marriage Problem with Master Preference Lists." Discrete Applied Mathematics 156:15 (2008), 2959-2977.

[Kavitha and Shah 06] T. Kavitha and C. Shah. "Efficient Algorithms for Weighted Rank-Maximal Matchings and Related Problems." In Algorithms and Computation: 17th International Symposium, ISAAC 2006, Kolkata, India, December 18-20, 2006, Proceedings, Lecture Notes in Computer Science 4288, pp. 153-162. New York: Springer, 2006.

[Lebedev et al. 07] D. Lebedev, F. Mathieu, L. Viennot, A.-T. Gai, J. Reynier, and F. de Montgolfier. "On Using Matching Theory to Understand P2P Network Design." Paper presented at the International Network Optimization Conference, Spa, Belgium, April 22-25, 2007.

[Lovász and Plummer 86] L. Lovász and M. D. Plummer. Matching Theory, Annals of Discrete Mathematics 29. Amsterdam: North-Holland, 1986.

[Manlove and O'Malley 05] D. F. Manlove and G. O'Malley. "Student Project Allocation with Preferences over Projects." In Proceedings of ACiD 2005: The 1st Algorithms and Complexity in Durham Workshop, Texts in Algorithmics 4, pp. 69-80. London: King's Publications, 2005.

[Manlove et al. 02] D. F. Manlove, R. W. Irving, K. Iwama, S. Miyazaki, and Y. Morita. "Hard Variants of Stable Marriage." Theoretical Computer Science 276:1-2 (2002), 261-279. 
[Mathieu 07a] F. Mathieu. "Self-Stabilization in Preference-Based Networks." In Proceedings of the 7th IEEE International Conference on Peer-to-Peer Computing, pp. 203-210. Los Alamitos, CA: IEEE Press, 2007.

[Mathieu 07b] F. Mathieu. "Upper Bounds for Stabilization in Acyclic PreferenceBased Systems." In Stabilization, Safety, and Security of Distributed Systems: 9th International Symposium, SSS 2007 Paris, France, November 14-16, 2007 Proceedings, Lecture Notes in Computer Science 4838, pp. 372-382. New York: Springer, 2007.

[Mehlhorn and Michail 05] K. Mehlhorn and D. Michail. "Network Problems with Non-polynomial Weights and Applications." Unpublished manuscript, 2005.

[Micali and Vazirani 80] S. Micali and V. V. Vazirani. "An $O(\sqrt{|V|} \cdot|E|)$ Algorithm for Finding Maximum Matching in General Graphs." In Proceedings of the 21st Annual IEEE Symposium on Foundations of Computer Science, pp. 17-27. Los Alamitos, CA: IEEE Press, 1980.

[O'Malley 07] G. O’Malley. "Algorithmic Aspects of Stable Matching Problems." PhD thesis, Department of Computing Science, University of Glasgow, 2007.

[Ronn 90] E. Ronn. "NP-Complete Stable Matching Problems." Journal of Algorithms 11 (1990), 285-304.

[Roth et al. 04] A. E. Roth, T. Sönmez, and M. U. Ünver. "Kidney Exchange." Quarterly Journal of Economics 119:2 (2004), 457-488.

[Roth et al. 05] A. E. Roth, T. Sönmez, and M. U. Ünver. "Pairwise Kidney Exchange." Journal of Economic Theory 125:2 (2005), 151-188.

[Scott 05] S. Scott. "A Study of Stable Marriage Problems with Ties." PhD thesis, Department of Computing Science, University of Glasgow, 2005.

[Sng 08] C. Sng. "Efficient Algorithms for Bipartite Matching Problems with Preferences." PhD thesis, Department of Computing Science, University of Glasgow, 2008 .

[Tan 91] J. J. M. Tan. "A Necessary and Sufficient Condition for the Existence of a Complete Stable Matching." J. Algorithms 12:1 (1991), 154-178.

David J. Abraham, Computer Science Department, Carnegie Mellon University, Wean Hall 4212, Pittsburgh, PA 15213-3891 (dabraham@cs.cmu.edu)

Ariel Levavi, Department of Computer Science and Engineering, University of California, San Diego, 9500 Gilman Drive, La Jolla, CA 92093-0404 (alevavi@cs.ucsd.edu)

David F. Manlove, Department of Computing Science, University of Glasgow, Glasgow, G12 8QQ, Scotland, UK (davidm@dcs.gla.ac.uk)

Gregg O'Malley, Department of Computing Science, University of Glasgow, Glasgow, G12 8QQ, Scotland, UK (gregg@dcs.gla.ac.uk)

Received March 29, 2008; accepted August 4, 2009. 